\title{
Optimal Bidding Strategies for Thermal and Combined Cycle Units in the Day-ahead Electricity Market with Bilateral Contracts
}

\author{
F. Javier Heredia, Marcos J. Rider, Member, IEEE, and Cristina Corchero.
}

\begin{abstract}
This paper developed a stochastic programming model that integrated the most recent regulation rules of the Spanish peninsular system for bilateral contracts in the dayahead optimal bid problem. Our model allows a price-taker generation company to decide the unit commitment of the thermal and combined cycle programming units, the economic dispatch of the $\mathrm{BC}$ between all the programming units and the optimal sale bid by observing the Spanish peninsular regulation. The model was solved using real data of a typical generation company and a set of scenarios for the Spanish market price. The results are reported and analyzed.
\end{abstract}

Index Terms-Electricity spot-market, short-term electricity generation planning, combined cycle units, bilateral contracts, optimal bidding strategies, stochastic programming.

\section{INTRODUCTION}

Generation companies in liberalized electricity markets do not have a load of their own to satisfy, but must bid their hourly generation to the market operator, who selects the lowestprice among the biding companies to match the pool load. A specific generation company (GenCo) expects to have most of its bids accepted, i.e., have them priced below the market price, determined hourly by matching the lowest-price bids with the pool load. Liberalized electricity markets are nowadays very sophisticated energy- and financial-transaction multimarkets where, around the main electricity market, the so-called "dayahead" or "spot" market, a portfolio of other financial and physical markets as well as bilateral contracts (BCs) exist. Moreover, a generation company operating in such a complex market can no longer optimize its medium- and short-term generation planning decisions without considering the relation between those markets and the increasing importance of the emission-free (wind power and hydro-generation) and lowemission technologies (combined cycle).

The BCs are agreements between a generation company and a qualified consumer to provide a given amount of electrical energy at a stipulated price along with a delivering period. The characteristics of the $\mathrm{BC}$ (energy, price and delivering period) are negotiated before the day-ahead market, either in organized or non-organized markets. In the organized $\mathrm{BC}$ markets, the

Manuscript send December 1, 2008. This work was supported by the Ministry of Science and Technology of Spain through CICYT Project DPI200509117-C02-01.

F. J. Heredia, M. J. Rider and C. Corchero are with the Group on Numerical Optimization and Modeling, Statistics and Operations Research Department, Universitat Politècnica de Catalunya, Edifici C5 Campus Nord, Jordi Girona 1-3, 08034, Barcelona, Spain.

e-mails: \{f.javier.heredia, marcos.rider, cristina.corchero\}@upc.edu

978-1-4244-4241-6/09/\$25.00 @2009 IEEE producers and consumers send sell and purchase bids that are matched by the market operator. Examples of organized BC markets are the CESUR [1] and EPE [2] auctions in Spain, BelpexVPP auctions in Belgium [3], and the EDF's capacity auctions in France [4]. In non-organized BC markets, the producers and consumers agree the amount, price, and period of the energy delivered, in a private negotiation. In both cases, from the point of the view of the generation company, a BC represents a scheduled load curve to be delivered, chargeable at a fixed price.

Several researchers have proposed optimal bidding models in the day-ahead market for thermal units under the pricetaker assumption, with or without BC. In [5] the authors presented a mixed-integer programming model to optimize the production schedule of a single unit with a simple bidding strategy. Furthermore, in [6], the approximation of step-wise bid curves by linear bid functions, based on the marginal costs was considered, although in a context without BC. Also, in [7] the concept of price-power function, which is similar to the matched energy function defined in our study, was used to derive the optimal bid curves of a hydro-thermal system. The mixed-integer stochastic programming model [8] distinguishes the variables corresponding to the bid energy and those representing the matched energy, though in a pricemaker framework and without BC. In [9] a stochastic unit commitment problem with $\mathrm{BC}$ was solved by maximizing the day-ahead market benefit. In [10] a mixed-integer stochastic optimization model for scheduling the thermal units was presented, and the production plants were optimized in the presence of stochastic market-clearing prices. Nevertheless, the two earlier models [9], [10] failed to propose any explicit modelization of the optimal bid as we have done. Furthermore, the general considerations about the bidding process in electricity markets can also be obtained [11]-[13].

The combined cycle (CC) units represent the majority of the new generating unit installations across the globe. In [14] a method was presented to calculate the unit commitment of $\mathrm{CC}$ units using dynamic programming and lagrangian relaxation applied to the security-constrained short-term scheduling problem. Furthermore, the price-based unit commitment problem based on the mixed-integer programming method for a generating company with thermal, CC, cascaded-hydro, and pumped-storage units has also been presented [15]. None of the earlier publications presented an explicit formulation of the optimal sale bid of the $\mathrm{CC}$ units to the day-ahead market or any considerations about the BC. 


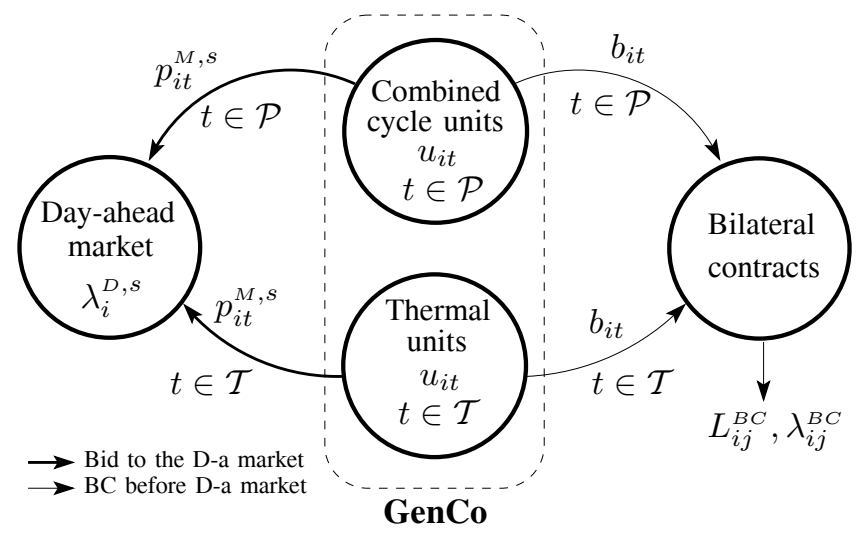

Figure 1. Representation of the system under study

Our study developed an stochastic mixed-integer quadratic programming model for a price-taker GenCo with $\mathrm{BC}$ obligations to determine the optimal bidding strategy of a pool of thermal and CC programming units in the day-ahead electricity market. The model was tested with real data of market prices and programming units of a GenCo operating in the Spanish electricity market [16].

\section{The Stochastic Programming Model}

Fig. 1 represents a price-taker GenCo possessing a set $\mathcal{T}$ of thermal units (coal, nuclear, fuel) and a set $\mathcal{C}$ of $\mathrm{CC}$ units represented by the associated set $\mathcal{P}$ of equivalent pseudo-units (combustion turbines and steam turbines, see section II-A below). Both thermal and CC units bid to the $i \in \mathcal{I}=\{1,2, \ldots, 24\}$ hourly auctions of the day-ahead market (left-oriented arrows of Fig. 1). The stochasticity of the spot price $\lambda_{i}^{D}, i \in \mathcal{I}$ is represented by a set of $\mathcal{S}$ scenarios. The set $\mathcal{B C}$ represents the portfolio of $\mathrm{BC}$ duties, with known energy $\left(L_{i j}^{B C} \mathrm{MWh}\right)$ and price $\left(\lambda_{i j}^{B C} € / \mathrm{MWh}\right)$ for each BC contract $j \in \mathcal{B C}$ and time period $i \in \mathcal{I}$, that must be dispatched between the thermal and $\mathrm{CC}$ units (right-oriented arrows of Fig. 1). The main information provided by the model (here and now decisions or first-stage variables) for each period, $i \in \mathcal{I}$, are the unit commitment of the thermal and CC units (variables $u_{i t}$ ), the energy allocated to the portfolio of bilateral contracts by each thermal and CC units (variables $b_{i t}$ ), and the optimal sale bid, expressed as a function of the previous firststage variables, which results in the second stage variables $p_{i t}^{M, s}$, the matched energy for scenario $s$.

\section{A. Thermal and Combined Cycle units operation}

The CC units represent a combination of combustion and steam turbines within a power plant. Typically, a CC unit consists of several combustion turbines (CTs) and an heat recovery steam generator and steam turbine (HRSG/ST) set. Based on the different combinations of CTs and HRSG/ST, a CC unit can operate at multiple states or configurations. The first two columns of Table I show the states of a CC unit with a CT and an HRSG/ST considered in this study. The operational rules of a typical CC unit were formulated [14] with the help of the so-called pseudo units (PUs). As the
Table I

STATES OF THE CC UNIT AND ITS ASSOCIATED PSEUdo UNITS

\begin{tabular}{cccccc}
\hline \multicolumn{7}{l}{ CC unit with a CT and HRSG/ST } & & & \\
\hline State & Composition & PU 1 & $u_{i \mathcal{P}_{c}(1)}$ & PU 2 & $u_{i \mathcal{P}_{c}(2)}$ \\
\hline 0 & $0 \mathrm{CT}+0 \mathrm{HRSG} / \mathrm{ST}$ & off & 0 & off & 0 \\
1 & $1 \mathrm{CT}+0 \mathrm{HRSG} / \mathrm{ST}$ & on & 1 & off & 0 \\
2 & $1 \mathrm{CT}+1 \mathrm{HRSG} / \mathrm{ST}$ & off & 0 & on & 1 \\
\hline
\end{tabular}

thermal units, the PUs of each CC unit have their own unique cost characteristics, real power generation limits, minimum on time limits, etc., and can be viewed as a special set of nonindependent or coupling single thermal units. The on/off state of these two PUs uniquely determined the state of the CC (see columns 3 and 5 of Table I), and allowed (as it will be seen later) a correct modelization of the operation of the state 0 without the need of an additional PU.

Let us define $\mathcal{P}_{c}$, the set of PUs of the $\mathrm{CC}$ unit $c \in \mathcal{C}$, and $\mathcal{P}=\cup_{c \in \mathcal{C}} \mathcal{P}_{c}$, the complete set of PUs. By $\mathcal{P}_{c}(j)$, we denote the PU associated with the state $j \in\{1,2\}$ of the CC unit $c$. Thus, $\mathcal{U}=\mathcal{T} \cup \mathcal{P}$ represents the complete set of units (thermal and pseudo). The on/off state of each thermal and pseudo units at period $i$ can be represented by the first-stage binary variables $u_{i t}, t \in \mathcal{U}$. Columns 4 and 6 of Table I illustrate the relation of the commitment binary variables of the PUs, $u_{i \mathcal{P}_{c}(1)}$ and $u_{i \mathcal{P}_{c}(2)}$, with the state of the associated CC unit.

However, the operation of each thermal unit must guarantee the minimum up $\left(t_{t}^{o n}\right)$ and down $\left(t_{t}^{o f f}\right)$ times. These conditions are introduced through the following set of constraints [17]:

$$
\begin{aligned}
& u_{i t}-u_{(i-1) t}-e_{i t}+a_{i t}=0 \\
& a_{i t}+\sum_{j=i}^{\min \left\{i+t_{t}^{\text {off }},|\mathcal{I}|\right\}} e_{j t} \leq 1 \\
& e_{i t}+\sum_{j=i+1}^{\min \left\{i+t_{t}^{o n},|\mathcal{I}|\right\}} a_{j t} \leq 1 \\
& u_{i t}, a_{i t}, e_{i t} \in\{0,1\} \cap \mathcal{K}_{t}
\end{aligned}
$$

where Eq. $(1 a)-(1 b)$ define the auxiliary binary variables $a_{i t}$ and $e_{i t}$ to be $a_{i t}=1$ iff $u_{(i-1) t}=1$ and $u_{i t}=0$, and $e_{i t}=1$ iff $u_{(i-1) t}=0$ and $u_{i t}=1$. The minimum up $\left(t_{t}^{o n}\right)$ and down $\left(t_{t}^{\text {off }}\right)$ times are guaranteed by Eq. $(1 b)-(1 c)$. The set $\mathcal{K}_{t}$ stands for the initial state of each unit. Analogously, each PU $t \in \mathcal{P}$ has its own minimum up time, $t_{t}^{o n}$ :

$$
\left.\begin{array}{ll}
u_{i t}-u_{(i-1) t}-e_{i t}+a_{i t}=0 \quad & (a) \\
e_{i t}+\sum_{j=i}^{\min \left\{i+t_{t}^{o n},|\mathcal{I}|\right\}} a_{j t} \leq 1 & (b) \\
u_{i t}, a_{i t}, e_{i t} \in\{0,1\} \cap \mathcal{K}_{t} &
\end{array}\right\} \begin{aligned}
& \forall i \in \mathcal{I} \\
& \forall t \in \mathcal{P}
\end{aligned}
$$

Each $\mathrm{CC}$ unit also has a minimum down time, i.e., once shut down, the CC unit cannot be started up before $t_{c}^{C}$ periods. The following constraints formulate the minimum down time condition for the CC units, expressed in terms of the binary variables of the $\mathrm{PU}$ of $\mathcal{P}_{c}$ with the aid of the feasible transition rules defined in Fig. 2: 


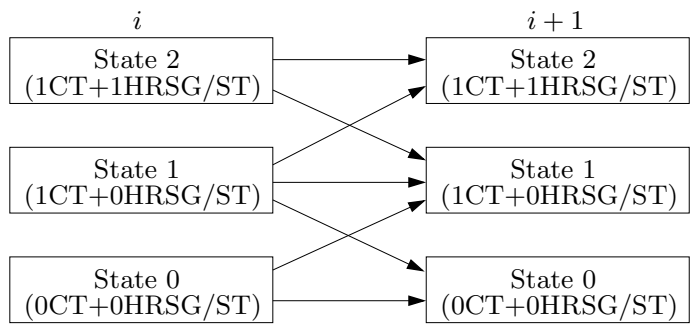

Figure 2. Feasible transitions of the $\mathrm{CC}$ unit with a CT and HRSG/ST

$$
\begin{aligned}
& \left(u_{i \mathcal{P}_{c}(1)}+u_{i \mathcal{P}_{c}(2)}\right)-\left(u_{(i-1) \mathcal{P}_{c}(1)}+u_{(i-1) \mathcal{P}_{c}(2)}\right)+ \\
& \left(a_{i \mathcal{P}_{c}(1)}-e_{i \mathcal{P}_{C}(1)}\right)-\left(e_{i \mathcal{P}_{c}(2)}-a_{i \mathcal{P}_{C}(2)}\right)=0 \\
& \left.\begin{array}{l}
\min \left\{i+t_{c}^{C},|\mathcal{I}|\right\} \\
\left.a_{i \mathcal{P}_{c}(1)}-e_{i \mathcal{P}_{c}(2)}\right)+\sum_{j=i} e_{j \mathcal{P}_{c}(1)}-a_{j \mathcal{P}_{c}(2)} \leq 1
\end{array}\right\} \begin{array}{l}
\forall i \in \mathcal{I} \\
\forall c \in \mathcal{C}
\end{array}
\end{aligned}
$$

with $\mathcal{K}_{t}^{C}$ standing for conditions imposed by the initial state of the $\mathrm{CC}$ unit. The feasible transitions rules impose additional constraints to the operation of the PUs associated to the same $\mathrm{CC}$ unit, $c \in \mathcal{C}$. First, the PUs in $\mathcal{P}_{c}$ are mutually exclusive (Eq. (4a)), i.e., only one of them can be committed at a given period (a CC can only be in one state simultaneously). Second, the change of the commitment of the PUs in $\mathcal{P}_{c}$ between periods $i$ and $i+1$ are limited to the feasible transitions depicted in Fig. 2. These feasible transitions impose that, if the CC unit $c$ is in state 0 at period $i\left(u_{i \mathcal{P}_{c}(1)}+u_{i \mathcal{P}_{c}(2)}=0\right)$, it cannot be in state 2 at period $i+1\left(u_{(i+1) \mathcal{P}_{c}(2)}=0\right)$ (Eq. $\left.(4 b)\right)$. Conversely, if $u_{i \mathcal{P}_{c}(2)}=1$, then $u_{(i+1) \mathcal{P}_{c}(1)}+u_{(i+1) \mathcal{P}_{c}(2)} \geq 1$ (Eq. (4c)). The following set of constraints formulates the specific operation rules of the $\mathrm{CC}$ units:

$$
\left.\begin{array}{ll}
\sum_{m \in \mathcal{P}_{c}} u_{i m} \leq 1 & (a) \\
u_{(i+1) \mathcal{P}_{c}(2)} \leq u_{i \mathcal{P}_{c}(1)}+u_{i \mathcal{P}_{c}(2)} & (b) \\
u_{i \mathcal{P}_{c}(2)} \leq u_{(i+1) \mathcal{P}_{c}(1)}+u_{(i+1) \mathcal{P}_{c}(2)} & (c)
\end{array}\right\} \begin{aligned}
& \forall i \in \mathcal{I} \\
& \forall c \in \mathcal{C}
\end{aligned}
$$

\section{B. Bilateral Contracts Constraint}

Consider that the GenCo has agreed to physically provide the energy amounts $L_{i j}^{B C}$ at hour $i \in \mathcal{I}$ of day D for each one of the $j \in \mathcal{B C}$ bilateral contracts. This energy $L_{i j}^{B C}$ can be provided by any programming unit $\mathcal{U}$, both thermal and PUs:

$$
\left.\begin{array}{l}
\sum_{t \in \mathcal{U}} b_{i t}=\sum_{j \in \mathcal{B C}} L_{i j}^{B C} \quad(a) \\
b_{i t} \in\left[0, \bar{p}_{t}\right] \quad \forall t \in \mathcal{U} \quad(b)
\end{array}\right\} \quad \forall i \in \mathcal{I}
$$

where the total contribution of the committed unit $t$ to the BC covering at period $i$ is represented by the variable $b_{i t}$.

\section{Optimal bid function and equivalent matched energy con- straints}

The optimal bid function could be developed under the the following assumptions, some of them being imposed by the MIBEL's rules: (a) The GenCo is a price-taker, i.e., the dayahead clearing price $\lambda_{i}^{D}$ does not depend on the GenCo's bidding; (b) The unit $t$ (thermal or PU) has been committed; (c) The unit $t$ would bid its minimum generation output $\underline{p}_{t}$ at zero price (instrumental bid); (d) The total contribution of unit $t$ to the BC covering must be excluded from the bid; and (e) The probability function of the clearing-price random variable $\lambda_{i}^{D}$ has been discretized in a set of scenarios $\mathcal{S}$ with associated clearing price $\lambda_{i}^{D, s}$ and probability $P^{s}, s \in \mathcal{S}$. By assuming the quadratic thermal generation costs, $C^{G}(p)=c_{t}^{b}+c_{t}^{l} p+c_{t}^{q}(p)^{2}$, the benefits obtained from the day-ahead market as a function of the matched energy $p_{i t}^{M, s}$ (the amount of energy that the thermal unit $t$ has to produce as a result of the marketclearing mechanism) for a given dispatched $\mathrm{BC}$ energy $b_{i t}$, under scenario $s$ will be:

$$
\begin{aligned}
B_{i t}^{D, s}\left(p_{i t}^{M, s}, b_{i t}\right)= & \lambda_{i}^{D, s} p_{i t}^{M, s}-\left[C^{G}\left(p_{i t}^{M, s}+b_{i t}\right)-C^{G}\left(b_{i t}\right)\right] \\
= & \lambda_{i}^{D, s} p_{i t}^{M, s}+ \\
& -c_{t}^{l} p_{i t}^{M, s}-c_{t}^{q}\left(p_{i t}^{M, s}\right)^{2}-2 c_{t}^{q} p_{i t}^{M, s} b_{i t}
\end{aligned}
$$

If assumptions (a)-(d) hold true, then it can be shown (Th. 1 of [18]) that the function :

$$
\lambda_{i t}^{B *}\left(p_{i t}^{B}, b_{i t}\right)=\left\{\begin{array}{l}
0 \quad \text { if } 0 \leq p_{i t}^{B} \leq\left[\underline{p}_{t}-b_{i t}\right]^{+} \\
\left.2 c_{t}^{q}\left(p_{i t}^{B}+b_{i t}\right)+c_{t}^{l} \text { if } \underline{p}_{t}-b_{i t}\right]^{+}<p_{i t}^{B} \leq \bar{p}_{t}-b_{i t} \\
\forall i \in \mathcal{I}, \forall t \in \mathcal{U}
\end{array}\right.
$$

with $[a-b]^{+}=\max \{0, a-b\}$, is the optimal bid function of unit $t$ for the day-ahead market $i$ in the sense that, for any given value $b_{i t}$, if function $\lambda_{i t}^{B *}$ is bid, the matched energy $p_{i t}^{M, s}$ corresponding to any scenario $s$ with market price $\lambda_{i}^{D, s}$, maximizes the day-ahead benefit function $B_{i t}^{D, s}$.

When $b_{i t}^{T}=0$ (the committed unit $t$ does not contribute to the $\mathrm{BC}$ covering), our problem reduces to the classical selfcommitment problem discussed by several authors [5], [6]. In this case, it is well known that the optimal-bid strategy for a price-taker GenCo consists of an instrumental bid up to the operational minimum limit, $\underline{p}_{t}$, and the rest of the plant capacity at the marginal price, $2 c_{t}^{q} p_{i t}+c_{t}^{l}$. This optimal policy corresponds to the particular case $b_{i t}=0$ of the generalized optimal-bid function $\lambda_{i t}^{B *}$. The matched energy for this particular case will be called the $B C$-free day-ahead matched energy, and can be represented by $p_{i t}^{D, s}$ :

$$
p_{i t}^{D, s} \equiv p_{i t}^{M, s}(0)=\left\{\begin{array}{lll}
\underline{p}_{t} & \text { if } \quad p_{i t}^{*, s} \leq \underline{p}_{t} & \forall i \in \mathcal{I} \\
p_{i t}^{*, s} & \text { if } \left.\quad p_{i t}^{*, s} \in\right] \underline{p}_{t}, \bar{p}_{t}[ & \forall t \in \mathcal{U} \\
\bar{p}_{t} & \text { if } \quad p_{i t}^{*, s} \geq \bar{p}_{t} & \forall s \in \mathcal{S}
\end{array}\right.
$$

with $p_{i t}^{*, s}=\left(\lambda_{i}^{D, s}-c_{t}^{l}\right) / 2 c_{t}^{q}$, the unconstrained minimum of the benefit function Eq. (6). $p_{i t}^{D, s}$ is a constant parameter of the model for a fixed thermal $t$, period $i$, and scenario $s$, and can be used to develop the expression of the optimal matched energy $p_{i t}^{M, s}\left(b_{i t}\right)[18]$ :

$$
p_{i t}^{M, s}\left(b_{i t}, u_{i t}\right)=\left\{\begin{array}{lll}
{\left[p_{i t}^{D, s}-b_{i t}\right]^{+}} & \text {if } u_{i t}=1 & \forall i \in \mathcal{I} \\
0 & \text { if } u_{i t}=0 & \forall t \in \mathcal{U} \\
& \forall s \in \mathcal{S}
\end{array}\right.
$$

Fig. 3 represents the function $p_{i t}^{M, s}\left(b_{i t}, u_{i t}\right)$ (thick line and dot). However, this non-differentiable expression cannot be included in the optimization model as it is. To formulate 


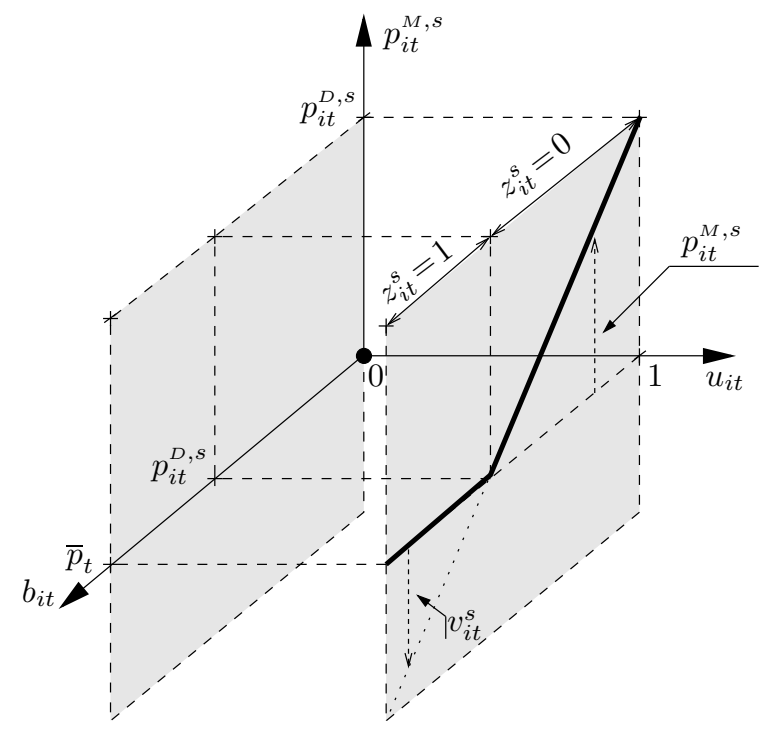

Figure 3. The matched energy function $p_{i t}^{M, s}$ for a fixed spot price $\lambda_{i}^{D, s}$

an equivalent mixed-integer linear formulation, we introduced the auxiliary binary $z_{i t}^{s}$ and continuous $v_{i t}^{s}$ variables (see Fig 3). In this formulation, $z_{i t}^{s}=1$ whenever $b_{i t} \geq p_{i t}^{D, s}$ and $z_{i t}^{s}=0$ otherwise, while $v_{i t}^{s}$ will be always defined as $v_{i t}^{s}=\left[b_{i t}-p_{i t}^{D, s}\right]^{+}$. With the help of these auxiliary variables, expression (8) can be transformed into the following equivalent mixed-integer linear system (Prop. 2 [18]):

$$
\begin{aligned}
& p_{i t}^{M, s}=p_{i t}^{D, s} u_{i t}+v_{i t}^{s}-b_{i t} \\
& p_{i t}^{D, s}\left(z_{i t}^{s}+u_{i t}-1\right) \leq b_{i t} \\
& b_{i t} \leq p_{i t}^{D, s}\left(1-z_{i t}^{s}\right)+\bar{p}_{t}\left(z_{i t}^{s}+u_{i t}-1\right) \\
& p_{i t}^{D, s}\left(1-z_{i t}^{s}\right) \geq p_{i t}^{M, s} \\
& p_{i t}^{D, s}\left(1-z_{i t}^{s}\right) \leq p_{i t}^{D, s} u_{i t} \\
& v_{i t}^{s} \leq\left(\bar{p}_{t}-p_{i t}^{D, s}\right)\left(z_{i t}^{s}+u_{i t}-1\right) \\
& \text { (e) } \forall i \in \mathcal{I} \\
& \text { (e) }\} \forall t \in \mathcal{U} \\
& p_{i t}^{M, s} \in\left[0, p_{i t}^{D, s}\right] \\
& \text { (f) } \forall s \in \mathcal{S} \\
& v_{i t}^{s} \in\left[0, \bar{p}_{t}-p_{i t}^{D, s}\right]
\end{aligned}
$$

The total generation output of thermal unit $t$ at each time period $i$ and scenario $s$ is given by:

$$
p_{i t}^{s}=p_{i t}^{M, s}+b_{i t} \quad \forall i \in \mathcal{I}, \forall t \in \mathcal{U}, \forall s \in \mathcal{S}
$$

\section{Objective function}

The expected value of the benefit function $B$ can be expressed as:

$$
\begin{aligned}
& \sum_{\lambda^{D}}\left[B\left(u, a, e, p, p^{M} ; \lambda^{D}\right)\right]= \\
& \sum_{\forall i \in \mathcal{I}} \sum_{\forall j \in \mathcal{B C}} \lambda_{i j}^{B C} L_{i j}^{B C} \\
& -\sum_{\forall i \in \mathcal{I}} \sum_{\forall t \in \mathcal{T}}\left[c_{t}^{o n} e_{i t}+c_{t}^{o f f} a_{i t}+c_{t}^{b} u_{i t}\right] \\
& -\sum_{\forall i \in \mathcal{I}} \sum_{\forall c \in \mathcal{C}}\left[c_{\mathcal{P}_{c}(1)}^{o n}\left(e_{i \mathcal{P}_{c}(1)}-a_{i \mathcal{P}_{c}(2)}\right)+c_{\mathcal{P}_{c}(2)}^{o n} e_{i \mathcal{P}_{c}(2)}\right]
\end{aligned}
$$

$$
\begin{aligned}
& -\sum_{\forall i \in \mathcal{I}} \sum_{\forall c \in \mathcal{C} \forall} \sum_{\forall t \in \mathcal{P}_{c}} c_{t}^{b} u_{i t} \\
& +\sum_{\forall i \in \mathcal{I}} \sum_{\forall t \in \mathcal{U}} \sum_{\forall s \in \mathcal{S}} P^{s}\left[\lambda_{i}^{D, s} p_{i t}^{M, s}-c_{t}^{l} p_{i t}^{s}-c_{t}^{q}\left(p_{i t}^{s}\right)^{2}\right]
\end{aligned}
$$

The term (11) is constant and corresponds to the BC profit. The term (12) is the on/off fixed cost of the unit commitment of the thermal units. The CC's start-up and fixed generation costs are formulated in term (13) and (14). In this formulation, as in [14], only start-up costs are associated to the PU, and no cost is associated to the transition from state 2 to state 1 . Both terms (12), (13) and (14) are deterministics and does not depend on the realization of the random variable $\lambda^{D}$. Finally, expression (15) represents the expected value of the benefit from the day-ahead market for thermal and CC units, where $P^{s}$ is the probability of scenario $s$. The generation cost functions of the PUs are modeled as quadratic functions as done in a couple of earlier studies [14], [15]. All the functions appearing in Eqs. (12) and (15) are linear except the generation costs in Eq. (15), which are concave quadratic $\left(c_{t}^{q} \geq 0\right)$.

\section{E. Final model}

The final model developed in the previous sections is as follows:

$\begin{cases}\max E_{\lambda^{D}}\left[B\left(u, a, e, p, p^{M} ; \lambda^{D}\right)\right] \\ \text { s.t. : } & \\ \text { Eq. }(1) & \text { Thermal unit commitment const. } \\ \text { Eq. }(2,3,4) & \mathrm{CC} \text { unit commitment const. } \\ \text { Eq. }(5) & \text { Bilateral contracts dispatching const. } \\ \text { Eq. (9) } & \text { Optimal matched energy const. } \\ \text { Eq. }(10) & \text { Definition of the total generation } p_{i t}^{s}\end{cases}$

The two-stage stochastic problem (16) corresponds to a mixed concave quadratic maximization problem with linear constraints.

\section{TEST AND RESULTS}

The model (16) has been tested using real data of a typical generation company and market prices for the Spanish Peninsular Electricity System [16] and the results are reported in this section. The day under study is Monday, May 05 2008, in the electricity market of mainland Spain. 3 bilateral contracts, 4 thermal units, 2 combined cycle units with a CT and a HRSG/ST and 24 hours of study were used in the tests. The characteristics of the thermal and $\mathrm{CC}$ units and $\mathrm{BCs}$ are shown in Table II, III and IV, respectively. The parameter $s t_{t}^{0}$ stands for the number of hours the unit has been on $\left(s t_{t}^{0}>0\right)$ or off $\left(s t_{t}^{0}<0\right)$ previous to the first optimization period. The minimum off time for both CC units (parameter $t_{c}^{C}$ in Eq. 3) was set to 3 hours, and also both CC units was considered shutdown for 3 hours previous to the first optimization period. The model (16) has been implemented in AMPL [19] and solved with CPLEX [20] (default options) using a SunFire X2200 with two dual core AMD Opteron 2222 processors at $3 \mathrm{GHz}$ and $32 \mathrm{~Gb}$ of RAM memory.

In order to test the two-stage stochastic model (16) a characterization of the market price $\lambda^{D}$ through a set of scenarios is required, where each scenario $\lambda^{D, s}=\left[\lambda_{1}^{D, s}, \ldots, \lambda_{24}^{D, s}\right]$ is 
Table II

OPERATIONAL CHARACTERISTICS OF THE THERMAL UNITS

\begin{tabular}{llllll}
\hline$t$ & $c_{t}^{b}$ & $c_{t}^{l}$ & $c_{t}^{q}$ & $\underline{p}_{t}$ & $\bar{p}_{t}$ \\
& $€$ & $€ / \mathrm{MWh}$ & $€ / \mathrm{MWh}^{2}$ & $\mathrm{MW}$ & $\mathrm{MW}$ \\
\hline 1 & 151.08 & 40.37 & 0.015 & 160.0 & 350.0 \\
2 & 554.21 & 36.50 & 0.023 & 250.0 & 563.2 \\
3 & 327.02 & 28.85 & 0.036 & 160.0 & 370.7 \\
4 & 197.93 & 36.91 & 0.020 & 160.0 & 364.1 \\
\hline \hline \multirow{2}{*}{$t$} & $s t_{t}^{0}$ & $c_{t}^{\text {on }}$ & $c_{t}^{\text {off }}$ & $t_{t}^{\text {on }}$ & $t_{t}^{\text {off }}$ \\
& $\mathrm{hr}$ & $€$ & $€$ & $\mathrm{hr}$ & $\mathrm{hr}$ \\
\hline 1 & +3 & 412.80 & 412.80 & 3 & 3 \\
2 & +3 & 803.75 & 803.75 & 3 & 3 \\
3 & -2 & 438.40 & 438.40 & 3 & 3 \\
4 & -1 & 419.20 & 419.20 & 3 & 3 \\
\hline
\end{tabular}

Table III

Operational Characteristics of The Combined CyCle Units

\begin{tabular}{llllll}
\hline$c$ & $\mathcal{P}_{c}$ & $\begin{array}{c}c_{t}^{b} \\
€\end{array}$ & $\begin{array}{l}c_{t}^{l} \\
€ / \mathrm{MWh}\end{array}$ & $\begin{array}{l}c_{t}^{q} \\
€ / \mathrm{MWh}^{2}\end{array}$ & $\begin{array}{l}\underline{p}_{t} \\
\mathrm{MW}\end{array}$ \\
\hline 1 & 5 & 151.08 & 50.37 & 0.023 & 160.0 \\
1 & 6 & 224.21 & 32.50 & 0.035 & 250.0 \\
2 & 7 & 163.11 & 55.58 & 0.019 & 90.0 \\
2 & 8 & 245.32 & 31.10 & 0.022 & 220.0 \\
\hline \hline$c$ & $\mathcal{P}_{c}$ & $\bar{p}_{t}$ & $s t_{t}^{0}$ & $c_{t}^{\text {on }}$ & $t_{t}^{\text {on }}$ \\
& & $\mathrm{MW}$ & $\mathrm{hr}$ & $€$ & $\mathrm{hr}$ \\
\hline 1 & 5 & 350.0 & -2 & 803.75 & 2 \\
1 & 6 & 563.2 & -2 & 412.80 & 2 \\
2 & 7 & 350.0 & -2 & 320.50 & 2 \\
2 & 8 & 700.0 & -2 & 510.83 & 2 \\
\hline
\end{tabular}

composed by a set of 24 hourly market prices. A set of 25 scenarios has been used, obtained as the result of the application of a scenario reduction algorithm [21] to the complete set of history data available from June 2007 to the day under study. A summary of the characteristics of the optimization problem and its solution is shown in Table V.

The optimal unit commitment of thermal and CC units is shown in Fig. 4. The three states or configurations of the $\mathrm{CC}$ units are represented as white (state 0 ), gray (state 1 , $\mathcal{P}_{c}(1)$ ) and black (state $2, \mathcal{P}_{c}(2)$ ) hourly blocks. Notice how the operation of the $\mathrm{CC}$ units obey the minimum up time and the feasible transition rules expressed by Eq. (2) and Eq. (4) respectively. When started-up, both $\mathrm{CC}$ units stay in state 1 longer than the minimum on time $t_{t}^{o n}=2$ before switching to the state 2 .

Table IV

Characteristics of the Bilateral Contracts

\begin{tabular}{lll}
\hline$j$ & $\begin{array}{l}L_{j=1 \ldots 24}^{B C} \\
\text { MW }\end{array}$ & $\begin{array}{l}\lambda_{j=1 \ldots 24}^{B C} \\
€ / \mathrm{MWh}\end{array}$ \\
\hline 1 & 200 & 75 \\
2 & 150 & 73 \\
3 & 250 & 78 \\
\hline
\end{tabular}

Table V

OPTIMIZATION CHARACTERISTICS OF THE STUDY CASE

\begin{tabular}{lllll}
\hline Constraints & $\begin{array}{l}\text { Real } \\
\text { variables }\end{array}$ & $\begin{array}{l}\text { Binary } \\
\text { variables }\end{array}$ & $\begin{array}{l}\text { E(Benefits) } \\
€\end{array}$ & $\begin{array}{l}\text { CPU } \\
\text { s }\end{array}$ \\
\hline 31927 & 9915 & 5240 & 850.058 & 893 \\
\hline
\end{tabular}

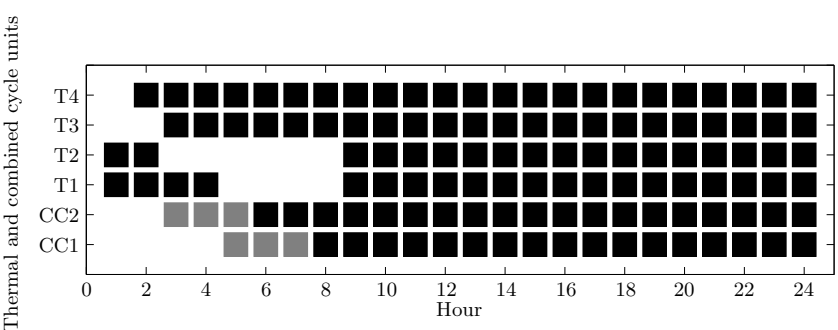

Figure 4. The unit commitment of thermal and $\mathrm{CC}$ units.

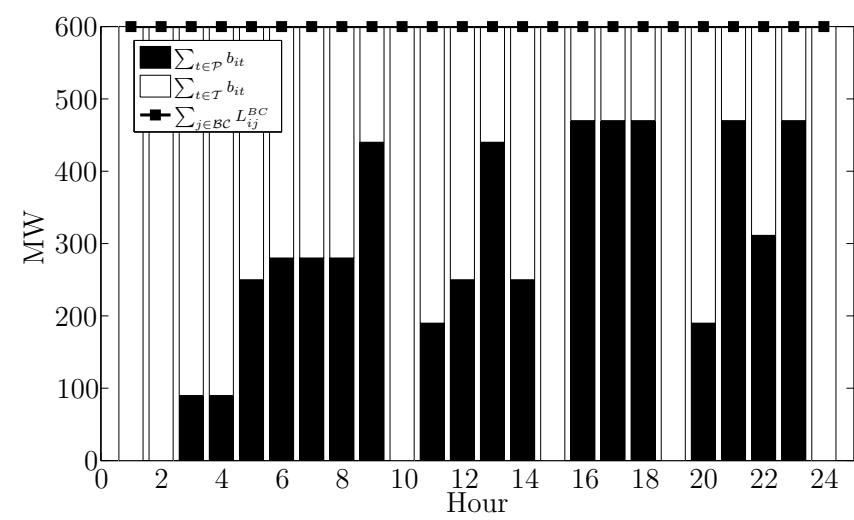

Figure 5. Management of the bilateral contracts $\left(\sum_{j \in \mathcal{B C}} L_{i j}^{B C}=600 \mathrm{MW}\right)$ between thermal and combined cycle units.

Fig. 5 shows the aggregated economic dispatch of the three BCs (600MWh) by the thermal (white bars) and the CC (black bars) units. It can be observed that, depending on the period, the portfolio of $\mathrm{BC}$ is covered exclusively by the thermal units (periods 1,2,10,15,19 and 24), or by a combination of thermal and $\mathrm{CC}$ units (the rest of the periods).

The optimal bid functions $\lambda_{i t}^{B *}$ for the thermal and CC units are represented in Fig. 6 and Fig. 7 respectively, where $b_{i \ldots k}$ represents the value of $b_{i t}$ at the different periods $i$, and $b_{*}$ corresponds to the rest of periods not explicitly indicated. To help the understanding of this graphics, let us analyze the
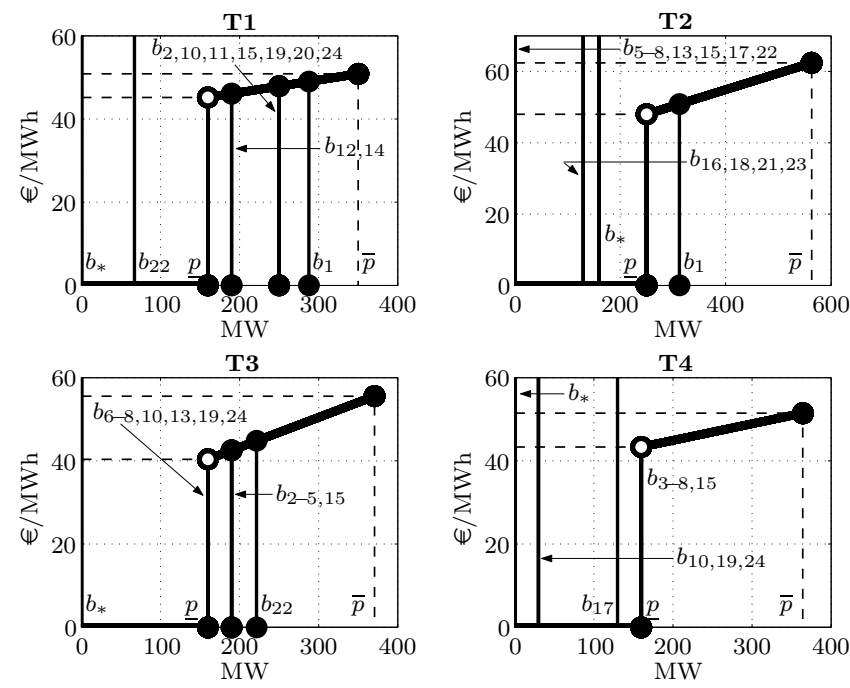

Figure 6. Representation of the optimal bid function $\lambda_{i t}^{B *}\left(p_{i t}^{B}, b_{i t}\right)$ of the thermal units. 

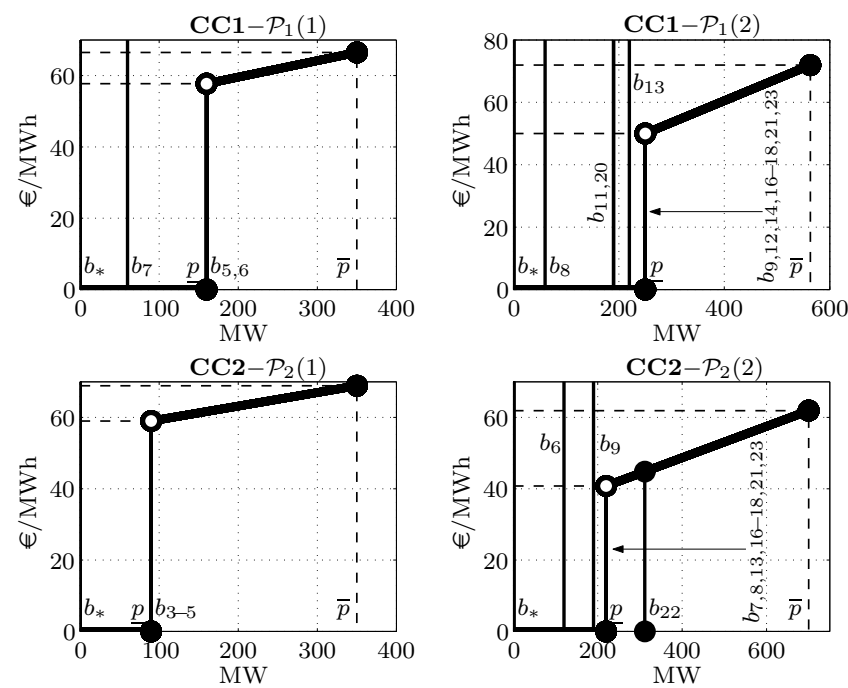

Figure 7. Representation of the optimal bid function $\lambda_{i t}^{B *}\left(p_{i t}^{B}, b_{i t}\right)$ of the $\mathrm{CC}$ units.

most simple case, the thermal unit $\mathrm{T} 4$, which is committed all the periods, except the first one. First, observe the piecewise discontinuous thick line, with a first block going from 0 to the minimum output $\underline{p}$, and a second block between $\underline{p}$ and $\bar{p}$, with a slope equal to the marginal cost of the thermal unit $2 c_{4}^{q}$. Both blocks correspond, respectively, to the two blocks defining the optimal bid function $\lambda_{i t}^{B *}$. After the development of Section II-C we know that this thick line represents the optimal bid function only in those periods where $b_{i t}=0$, (periods $i \in\{2,9,11-14,16,18,20-23\}$ for the thermal unit T4). Moreover, for those periods where $b_{i t}>0$, the optimal bid function corresponds to the part of the thick line between the auxiliary second vertical axis shown in Fig. 6 located at $b_{i t}$, and $\bar{p}_{t}$. In the case of the thermal unit T4, $b_{i, 4}=\underline{p}_{4}=160 \mathrm{MW}$ for periods $i \in\{3-8,15\}, b_{i, 4}=130 \mathrm{MW}$ for period $i=17$ and $b_{i, 4}=30 \mathrm{MW}$ for periods $i \in\{10,19,24\}$. Although $b_{i, 4} \leq \underline{p}_{4} \forall i$ in the case of the thermal unit T4, this could not be the case for other thermal units: see for instance the optimal bid function of thermal unit T3, where $b_{i, 3}=190 \mathrm{MW}$ $\forall i \in\{2-5,15\}$ and $b_{22,3}=221.5 \mathrm{MW}$, both values above the minimum generation $\underline{p}_{3}=160 \mathrm{MW}$. The optimal bid functions of the remaining thermal units of Fig. 6 can be interpreted in a similar way.

Let us now focus our attention on the optimal bid functions of the CC units (Fig. 7). First observe how each CC has two different sets of optimal bid functions, depending on the state of the $\mathrm{CC}$ unit at each period $i$. The $\mathrm{CC}$ unit 1 would send the optimal bid functions $\mathrm{CC} 1-\mathcal{P}_{1}(1)$ at periods 5,6 and 7 , where this CC unit is in state 1 (gray blocks of Fig. 4), and the optimal bid functions $\mathrm{CC} 1-\mathcal{P}_{1}(2)$ at the rest of the periods (black blocks of Fig. 4). The same happens with the second CC unit, CC2. Please notice that the optimal bid function of each state of the same $\mathrm{CC}$ unit has its own slope, which corresponds to the marginal cost of each PU.

\section{CONCLUSIONS}

A two-stage stochastic mixed-quadratic programming problem has been proposed to decide the optimal unit commitment and sale bid to the day-ahead market, and the optimal economic dispatch of the bilateral contracts for all the thermal and combined cycle units observing the MIBEL regulation. The model was implemented and solved with commercial optimization packages and tested with real data of a Spanish generation company and market prices. The results of the computational experiments show the validity of the presented model and its applicability to real problems.

\section{REFERENCES}

[1] (2008) Spain CESUR bilateral contracts auctions. CESUR. [Online] Available: http://www.subastascesur.omel.es/.

[2] (2008) Spain Virtual Power Plant Auctions. MEFF. [Online]. Available: https://www.subasta-epe.com/.

[3] (2008) Belgium Virtual Power Plant Auctions. [Online]. Available: http://www.belpexvpp.be/.

[4] (2008) Electricité de France Capacity Auctions. [Online]. Available: http://capacityauctions.edf.fr/.

[5] A. J. Conejo, F. J. Nogales and J. M. Arroyo, "Price-taker bidding strategy under price uncertainty," IEEE Trans. on Power Syst., vol. 17, no. 4, pp. 1081-1088, Nov. 2002.

[6] V. P. Gountis, A. G. Bakirtzis, "Bidding Strategies for Electricity Producers in a Competitive Electricity Marketplace," IEEE Trans. on Power Syst., vol. 19, no. 1, pp. 356-365, Feb. 2004.

[7] E. Ni, P. B. Luh and S. Rourke, "Optimal Integrated Generation Bidding and Schedulling With Risk Management Under a Deregulated Power Market," IEEE Trans. on Power Syst., vol. 19, no. 1, pp. 600-609, Feb. 2004.

[8] M. Nowak, R. Schultz and M. Westphalen, "A Stochastic Integer Programming Model for Incorporating Day-Ahead TRading of Electricity into Hydro-Thermal Unit Commitment," Optimization and Engineering, vol. 6, pp. 163-176, 2005.

[9] G. B. Shresta, S. Kai and L. Goel, "An efficient stochastic selfscheduling technique for power producers in th ederegulated power market," Electric Power Systems Research, vol. 71, pp. 91-98, 2004.

[10] C. Triki, P. Beraldi and G. Gross, "Optimal capacity allocation in multiauction electricity markets under uncertainty," Computers \& Operations Research, vol. 32, no. 2, pp. 201-217, 2005.

[11] E. J. Anderson and A. B. Philpott, "Optimal offer construction in electricity markets," Mathematics of Operations Research, vol. 27, no. 1, pp. 82-100, 2002.

[12] E. J. Anderson and A. B. Philpott, "Using supply functions for offering market generation into an electricity market," Operations Research, vol. 50, no. 3, pp. 477-489, 2003.

[13] P. Neame, A. B. Philpott and G. Pritchard, "Offer stack optimisation in electricity pool markets," Operations Research, vol. 51, no. 3, pp. 397-408, 2003.

[14] B. Lu and M. Shahidehpour, "Short-term scheduling of combined cycle units," IEEE Trans. on Power Syst., vol. 19, no. 3, pp. 1616-1625, Aug. 2004.

[15] T. Li and M. Shahidehpour, "Price-based unit commitment: A case of lagrangian relaxation versus mixed integer programming," IEEE Trans. on Power Syst., vol. 20, no. 4, pp. 2015-2025, Nov. 2005.

[16] (2008) Market Operator of the Electricity Market of Mainland Spain. OMEL. [Online]. Available: http://www.omel.es/.

[17] N. Nabona and A. Pages, "A three-stage short-term electric power planning procedure for a generation company in a liberalized market," International Journal of Electric Power Systems, vol. 29, no. 5, pp. 408421, 2007.

[18] F. J. Heredia, M. J. Rider and C. Corchero, "A stochastic programming model for the optimal electricity market bid problem with bilateral contracts for thermal and Combined Cycle units," Technical Report, Technical University of Catalonia, pp. 18, E-prints UPC http://hdl.handle.net/2117/2282, Oct. 2008.

[19] R. Fourer, D. M. Gay and B. W. Kernighan, AMPL: A modeling language for mathematical programming. CA: Brooks/Cole-Thomson Learning, Pacific Grove, 2nd Ed., 2003.

[20] "CPLEX Optimization subroutine library guide and reference, version 11.0," CPLEX Division, ILOG Inc., Incline Village, NV, USA, 2008.

[21] N. Gröwe-Kuska, H. Heitsch and W. Römisch, "Scenario reduction and scenario tree construction for power management problems," in Proc. IEEE PowerTech, vol. 3, Bologna, Italy, 2003. 